\title{
Probing the Sea Quark Content of the Proton with One-Particle-Inclusive Processes
}

\author{
Ignacio Borsa ${ }^{*}$ and Rodolfo Sassot \\ Departamento de Física and IFIBA, Facultad de Ciencias Exactas y Naturales, \\ Universidad de Buenos Aires, Ciudad Universitaria, Pabellón 1 (1428) Buenos Aires, Argentina \\ Marco Stratmann $]^{\ddagger}$ \\ Institute for Theoretical Physics, University of Tübingen, \\ Auf der Morgenstelle 14, 72076 Tübingen, Germany
}

\begin{abstract}
We investigate the feasibility of constraining parton distribution functions in the proton through a comparison with data on semi-inclusive deep-inelastic lepton-nucleon scattering. Specifically, we reweight replicas of these distributions according to how well they reproduce recent, very precise charged kaon multiplicity measurements and analyze how this procedure optimizes the determination of the sea quark densities and improves their uncertainties. The results can help to shed new light on the long standing question on the size of the flavor and charge symmetry breaking among quarks of radiative origin. An iterative method is proposed and adopted to account for the inevitable correlation with what is assumed about the parton-to-hadron fragmentation functions in the reweighting procedure. It is shown how the fragmentation functions can be optimized simultaneously in each step of the iteration. As a first case study, we implement this method to analyze kaon production data.
\end{abstract}

\section{INTRODUCTION AND MOTIVATION}

Parton distribution functions (PDFs) [1] are an essential ingredient in the description of nucleons as needed in the quantitative interpretation of hadronic hard scattering processes in the framework of perturbative Quantum Chromodynamics (pQCD). Based on the notion of factorization and together with the hadronization "probabilities" of produced final-state partons into observed hadrons, commonly known as fragmentation functions (FFs) [2], PDFs encode all the non-perturbative details about the hadronic structure and the path into confinement relevant in theoretical calculations of hard interactions.

The growing need to validate increasingly precise nonperturbative approaches aimed at describing the partonic structure of nucleons [3, 4], as well as the requirement of having available unprecedentedly accurate predictions for a multitude of hadronic processes where the Standard Model and possible extensions thereof can be challenged, place the pursuit of acquiring a precise knowledge on PDFs right the center of the most topical discussions at present [1].

In spite of the strenuous efforts and the remarkable degree of sophistication achieved in the extraction of PDFs and the quantification of their uncertainties in the last two decades [5], the PDFs of largely radiative origin in the nucleon, namely the quark-antiquark pairs produced by gluon splitting, still lack the desired precision. The results for sets of PDFs obtained in global fits to data by the currently most active collaborations may differ, for

\footnotetext{
*Electronic address: borsa.ignacio@gmail.com

$\dagger$ Electronic address: sassot@df.uba.ar

${ }^{\ddagger}$ Electronic address: marco.stratmann@uni-tuebingen.de
}

instance, by up to forty percent for strange quarks and by up to ten percent for the lighter sea quarks [1].

In addition, experimental results with a particular, albeit indirect sensitivity to sea quark densities of a certain flavor, such as measurements of the leptonic decays of $\mathrm{W}$ bosons at the CERN-LHC [6] 8$]$, electroweak charged-current deep-inelastic scattering (DIS) experiments [9 12], and kaon multiplicities in semi-inclusive deep-inelastic scattering (SIDIS) [13] seem to suggest divergent answers for the shape and normalization of the strange quark PDF. More specifically, while (SI)DIS experiments favor a sizable suppression of strange quarks relative to non-strange antiquarks in the proton of the order of $50 \%$, ATLAS results favor scenarios with almost no or very little suppression, while CMS prefers a suppressed strange sea.

In the most naive approximation, one would expect a charge and $\mathrm{SU}(3)$ flavor symmetric population of quarks of radiative origin, since they are mainly produced by gluon splitting into quark and antiquark pairs, which is flavor blind. However, different degrees of breaking of these symmetries have been found in various experiments, albeit within very large uncertainties. Several competing mechanisms have been proposed in the literature 14 to explain or motivate such findings but none of them seems to be conclusive.

The main difficulties inherent to fully disentangling the share of different flavors in the proton were noticed already in the very early days of the parton model [15], as well as a plausible solution: utilizing the rich information coming from the SIDIS production of hadrons with a different flavor content. For instance, charged kaon multiplicities obtained off proton targets at medium to large parton momentum fractions $x$ contain more positively charged kaons than negative ones according to the relative abundances of $u$ and $\bar{u}$ quarks in the proton, and also critically depend on their ratio to strange quarks. 
The recent access to precisely measured multiplicities in SIDIS for positively and negatively charged pions and kaons, produced alternatively off proton and deuteron targets, and within different regions of momentum fraction $x$ [13, 16], allows, in principle, to study the flavor dependence of PDFs and FFs at an unprecedented level of accuracy. The main caveat of such an approach is that it requires a simultaneous extraction of PDFs and FFs, with the cumbersome requirement consisting of the $\chi^{2}$ minimization of a very large number of correlated parameters in a global QCD fit. As a first step in that direction, we explore in what follows a sequential, iterative approach, where one-particle-inclusive measurements are used first to refine existing sets of PDFs that are in turn adopted as the ingredient in a determination of FFs, subsequently utilized in obtaining a next generation of PDFs. In the present paper, this procedure is implemented and adopted for kaon production data. As we shall discuss in detail, the method is found to converge very fast to new optimum sets of PDFs and FFs that provide a more accurate description of the data along with a noticeable reduction in the uncertainties.

A key ingredient in the above mentioned approach is the so-called reweighting technique for PDFs as developed and heavily used by the NNPDF collaboration [17, 18]. This strategy allows one to incorporate retroactively but consistently the information contained in data sets that are not included in the original global extraction of PDFs and in the determinations of their uncertainties. In particular, this framework avoids a full and time-consuming re-fit but preserves the statistical rigor of the original extraction. The method has already been demonstrated in different applications, see, e.g., [17 20].

The remainder of the paper is organized as follows: in the next section we briefly recall the status of the pQCD description of kaon production in SIDIS, the recent experimental findings, how they constrain kaon FFs, and how much room is left for refining the PDF set adopted in the calculation. Next, we sketch the main features of the PDFs reweighting technique and how it applies to the case under consideration. In Section III, we discuss in some detail the outcome of our reweighting exercise for kaon production and the iterative strategy outlined above and compare the effects of adopting different sets FFs as the starting point of our analyses. We briefly summarize the main results in Section IV.

\section{ROLE OF SIDIS DATA IN GLOBAL FITS}

\section{A. Status of FFs extractions using SIDIS data}

Recently, the COMPASs experiment at CERN 16 published extremely precise SIDIS multiplicity measurements for charged kaons produced off deuteron targets. The data are presented with a rather fine binning in the relevant kinematical variables. Together with the multiplicity results from the HERMES experiment at DESY
[13] taken off both proton and deuteron targets at smaller transferred momentum $Q$ than the COMPASS results though, the new data allowed for a very precise determination of charged kaon FFs and, for the first time, also their uncertainties in a global QCD analysis [22], that supersedes a previous, less sophisticated extraction performed almost ten years ago [23]. The global fits [22, 23] incorporate also additional information from other nonSIDIS experiments, semi-inclusive electron-position annihilation (SIA) and inclusive hadron production at high transverse momentum $p_{T}$ in hadron-hadron collisions, which constrained mainly the total flavor singlet FF and the gluon FF, respectively [22, 23]. The specific and unique role of SIDIS data in determining flavor and charge separated FFs was demonstrated in 22].

Kaon multiplicities $M_{l p(l d)}^{K^{ \pm}}$in lepton-proton $(l p)$ or lepton-deuteron $(l d)$ scattering are defined as the ratio of the inclusive charged kaon yield in SIDIS and the total DIS cross section in the same kinematic bins of parton momentum fraction $x$ and photon virtuality $Q^{2}$ :

$$
M_{l p(l d)}^{K^{ \pm}} \equiv \frac{d \sigma_{l p(l d)}^{K^{ \pm}} / d x d Q^{2} d z}{d \sigma_{l p(l d)} / d x d Q^{2}} .
$$

In the global fit 22] the two-dimensional projections of the three-dimensional multiplicity data [13] onto both the $z-Q^{2}$ and $z-x$ dependence are considered, for four different bins of the kaon's momentum fraction $z$ in the case of the Hermes data. Compass results are presented as a function of $z$ in 9 bins of $x$, each subdivided into various bins in the inelasticity parameter $y$ that effectively select different $Q^{2}$-ranges. In total 144 (309) data points for both $K^{+}$and $K^{-}$multiplicities are presented in the case of HERMES (COMPASs).

Non-SIDIS data comprise $K^{-} / K^{+}$and $K / \pi$ production ratios measured in proton-proton collisions by the STAR collaboration at BNL-RHIC [24] and the ALICE collaboration at the LHC 25] respectively, and SIA data from BELLE 26], BABAR 27] as well as some older LEP and SLAC experiments 28 32]. The combined use of data on different processes, such as SIA, SIDIS and proton-proton collisions, is essential to disentangle much more precisely the flavor, charge, and kinematical dependence of the hadronization probabilities. The so obtained FFs inherit ambiguities according to the experimental uncertainties and the precision of theory estimates for the different processes due to the choice of scales, PDFs, etc. These sources of uncertainties are duly included in the error estimates obtained in Ref. [22].

The overall agreement between the data and the theoretical calculations based on the DSS 17 set of FFs 22] at next-to-leading order (NLO) accuracy, quantified in terms of the usual $\chi^{2}$ per degree of freedom function, is remarkably good and significantly superior than the one achieved with the now outdated DSS 07 set of FFs [23]. A closer inspection reveals, however, that the theoretical description is far from being perfect in the case of quite some of the SIDIS data points, and it cannot be improved 


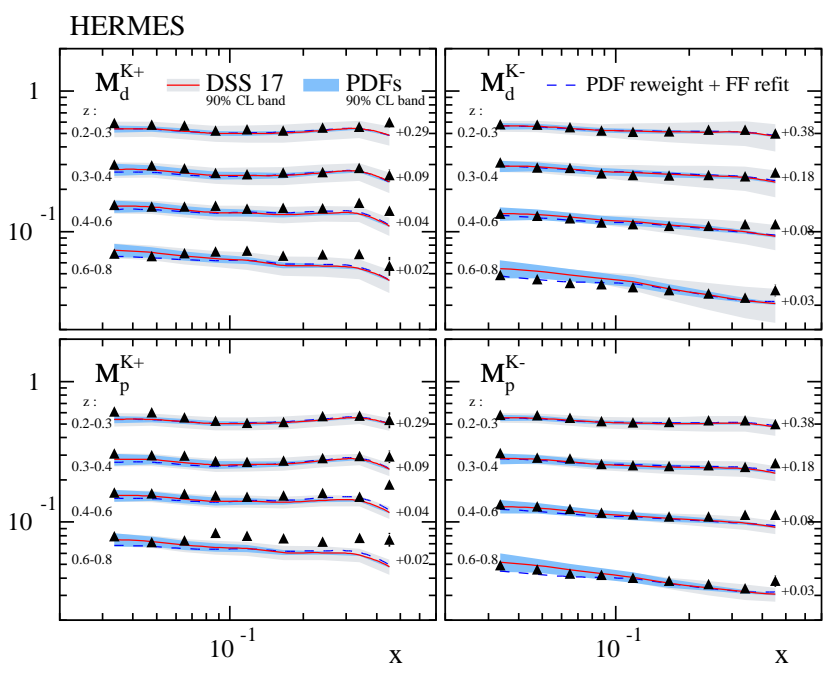

FIG. 1: NLO results for charged kaon multiplicities in SIDIS computed with the DSS 17 set of FFs 22] and the MMHT 14 set of PDFs 33] (solid lines) for both proton and deuteron targets compared to data from the HERMES collaboration [13]. The light shaded (grey) bands correspond to the uncertainty estimates for the FFs, and the dark shaded (blue) bands represent the uncertainties of the MMHT 14 set of PDFs, both at the $90 \%$ confidence level (C.L.). The dashed lines are the NLO results obtained with a reweighted set of PDFs and a refined set of FFs, see text.

further by allowing for either a greater flexibility in the functional parameter space chosen for the input FFs or by relaxing the standard assumptions about the charge or flavor symmetry of FFs. The overall $\chi^{2}$ of the SIDIS data in the DSS 17 analysis is nevertheless very much acceptable because of the rather large PDFs uncertainties in the relevant kinematic regime probed by HERMEs and COMPASs which are included as a theoretical uncertainty. Obviously, this raises the question whether the agreement with SIDIS data could be improved by fine-tuning the PDFs within their estimated, rather large uncertainties.

In Fig. 1 data on charged kaon multiplicities in SIDIS from the HERMES collaboration [13], taken on both proton and deuteron targets, are compared to NLO results computed with the DSS 17 set of FFs 22] and the MMHT 14 set of PDFs [33]. The shaded bands illustrate the respective uncertainty estimates for the FFs and PDFs. Roughly speaking, the uncertainty stemming from the chosen set of PDFs is larger for $K^{-}$than for $K^{+}$multiplicities mainly because of the $\bar{u} s$ content of the $K^{-}$meson that predominantly couples to corresponding $\bar{u}$ and $s$ sea quark PDFs compared to the $u \bar{s}$ content of the $K^{+}$meson. The $u$ quark PDF relevant for $K^{+}$ production is significantly better constrained than the $\bar{u}$ sea quark PDF. Because of this larger dependence on sea quark PDFs, $K^{-}$multiplicities are also typically smaller and fall off faster with increasing parton momentum fractions $x$ than their $K^{+}$counterparts. In both cases the
PDFs uncertainties grow towards smaller $x$ because of the relative enhancement of the sea quark content.

The larger the PDF uncertainties or the differences between data and theory estimates in Fig. 1, the greater is the potential to further constrain them with the SIDIS data. Indeed, anticipating the results of our iterative reweighting technique to be discussed in Sec. III below, the dashed lines in Fig. 1 correspond to NLO calculations utilizing reweighted sets of PDFs and a refined set of FFs that was obtained by using the so improved PDFs in the global analysis.

Figures 2 and 3 show the corresponding SIDIS multiplicities for charged kaons off a deuteron target as a function of the hadron momentum fraction $z$ as measured by COMPASS. Each panel corresponds to a different bin in $x$ and, consequently, probes the PDFs in a different kinematic region. Specifically, the first three panels correspond to $x$ values lower than those covered by the HERMES data shown in Fig. 1 Within each $x$-bin, the data are divided in bins of the inelasticity parameter $y$ that corresponds to different photon virtualities $Q^{2}$. Again, the data extend the range on $Q^{2}$ as covered by Hermes.

\section{B. PDFs reweighting for SIDIS data}

The reweighting technique allows one to incorporate consistently the information contained in new data into an existing set of PDFs without the need of refitting them but preserving the statistical rigor of its extraction [17, 18]. The method has already been successfully demonstrated in different applications. Here, we briefly recall its main features which are needed for our discussions below.

The method is based on statistical inference and starts with the generation of a large ensemble of PDF replicas $f_{k}$, each fitted to a data replica generated according the experimental uncertainties and correlations. The ensemble forms an accurate representation of the probability distribution of PDFs, and any quantity $\mathcal{O}$ depending on the PDFs can be evaluated by averaging the results for the individual replicas:

$$
\langle\mathcal{O}\rangle=\frac{1}{N} \sum_{k=1}^{N} \mathcal{O}\left[f_{k}\right],
$$

with $N$ the number of replicas. Using Bayesian inference it is possible to update the original probability distribution to a new one that accounts for the information contained in a new measurement, by assigning a new weight $w_{k} \neq 1$ for each replica, which measures its agreement with the new data. The updated estimate for any quantity then becomes

$$
\langle\mathcal{O}\rangle_{\text {new }}=\frac{1}{N} \sum_{k=1}^{N} w_{k} \mathcal{O}\left[f_{k}\right] .
$$

The Bayesian reweighting is fully equivalent to a full new fit provided the new data set is not too constraining, 


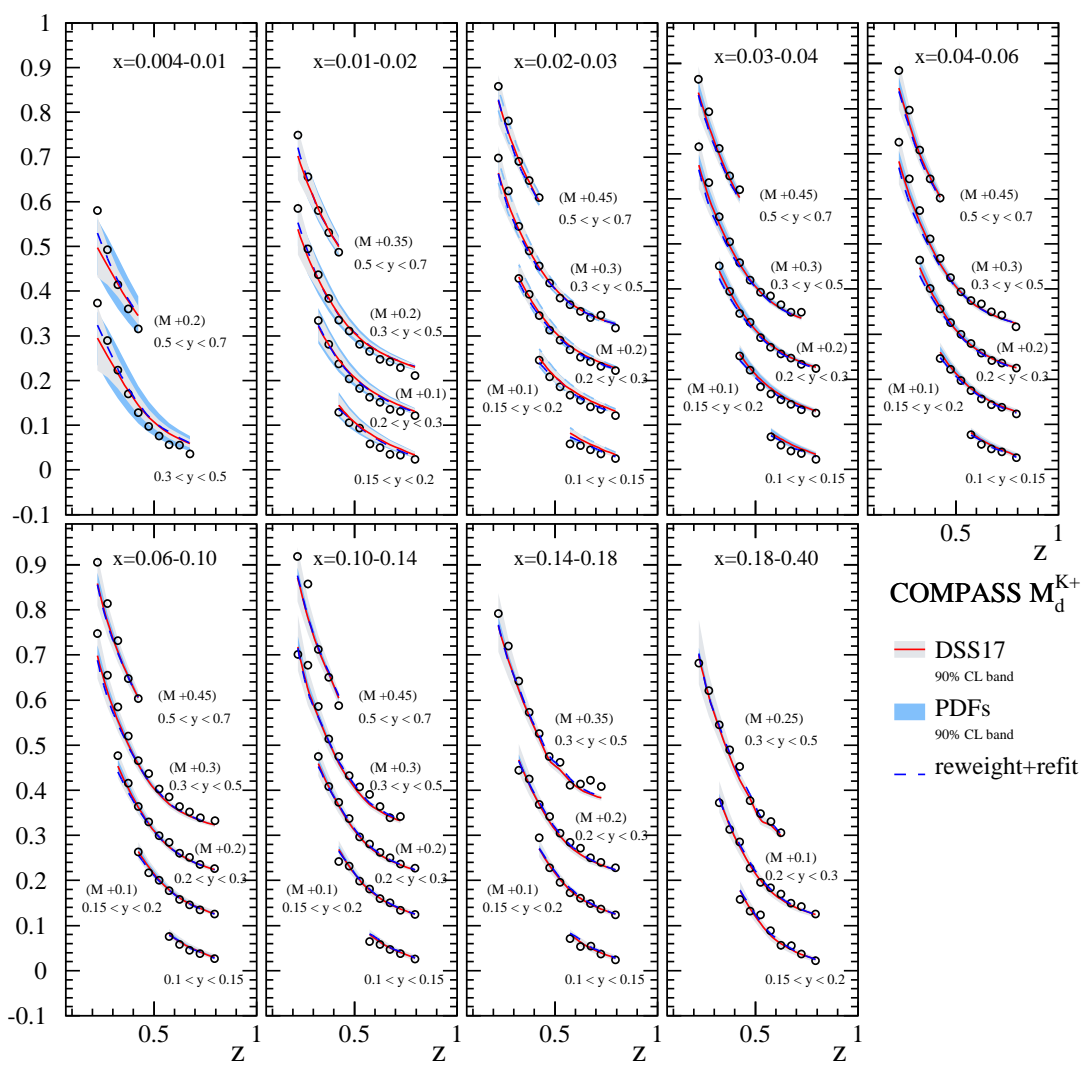

FIG. 2: Same as in Fig. 1 but now for the positively charged kaon multiplicities on a deuteron target obtained by Compass [16]; see text.

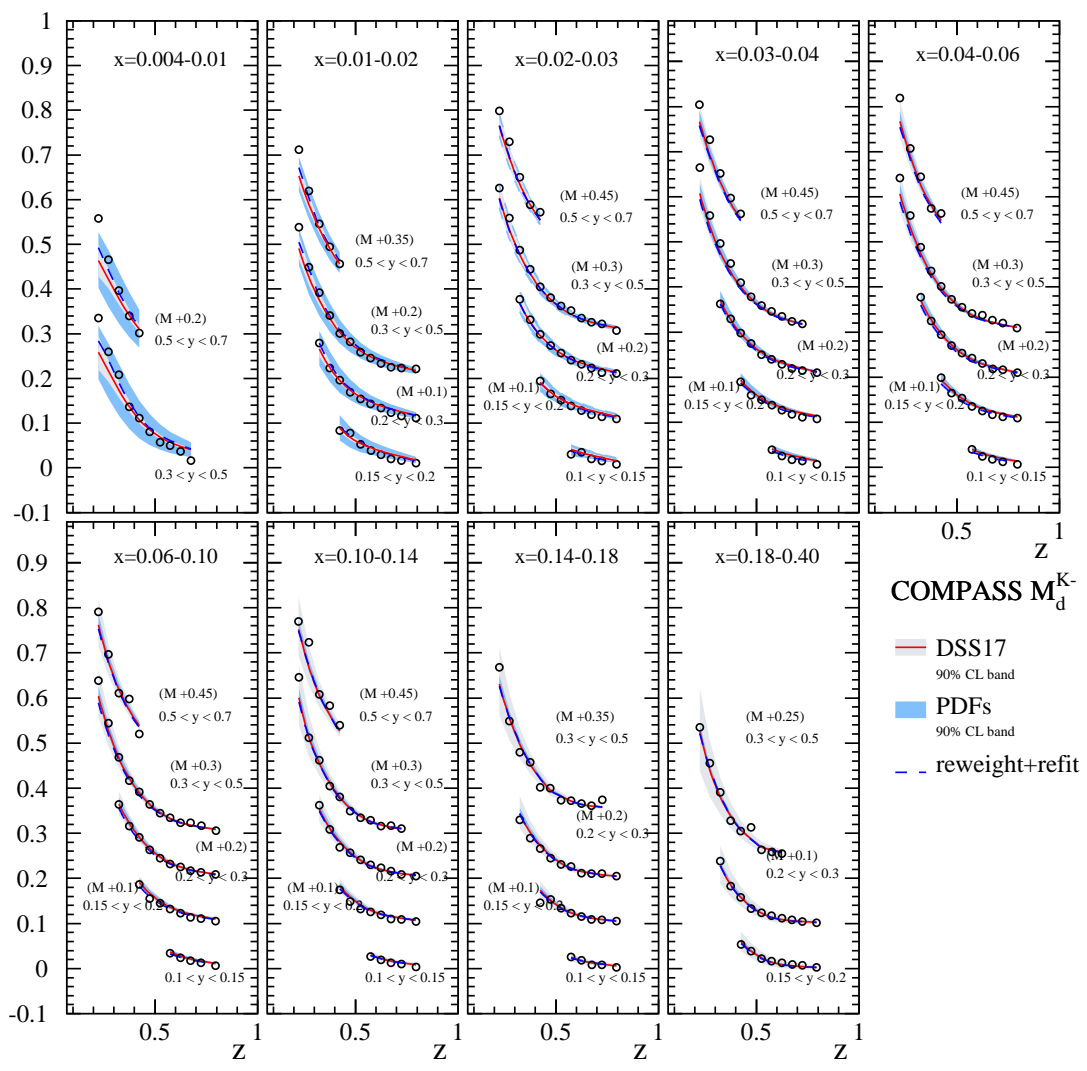

FIG. 3: Same as in Fig. 2 but for negatively charged kaons. 
so that the effective number of replicas, i.e. those with a non-negligible weight $w_{k}$, is still large enough. Two consistency checks are usually performed in order to verify that a new set of data can be satisfactorily described by a large enough number of replicas in the initial ensemble. The first one is the direct examination of the $\chi^{2}$ profile for the new set of data normalized by the number of data $N_{\text {data }}$ before and after the reweighting. Alternatively, it is possible to estimate by how much the agreement between data and theory improves by rescaling the uncertainties of the new data by a factor $\alpha$, whose probability is proportional to

$$
\mathcal{P}(\alpha) \propto \frac{1}{N} \sum_{k=1}^{N} w_{k}(\alpha) \mathcal{O}\left[f_{k}\right]
$$

If $\mathcal{P}(\alpha)$ is peaked near one, the new data is consistent with the initial theory distribution, while if it is peaked at a larger value, it may suggest that uncertainties have been underestimated or the underlying theory is not adequate to describe the newly included measurement.

The NNPDF collaboration provides an ensemble of 1000 replicas for its PDF set NNPDF 3.0 [21] which we utilize in the following to compute weights $w_{k}$ according to how well each replica $k$ reproduces the COMPASs and HERMES data for charged kaon production. The SIDIS multiplicities are computed at NLO accuracy by convoluting the recent DSS 17 set of parton-to-kaon FFs 22] with each of the NNPDF 3.0 replicas and the appropriate NLO coefficient functions as was described, for instance, in Refs. [22, 23].

The agreement between the theoretical estimate and data not only depends on the quoted experimental errors and on the uncertainties of the chosen set of PDFs but also on the ambiguities inherent to the set of parton-tokaon FFs used in the computation. Therefore, it seems reasonable to include also the uncertainty estimates on the FFs given in Ref. [22] in the $\chi^{2}$ estimate by propagating them to the SIDIS multiplicities. However, this procedure necessarily introduces some ambiguity: the uncertainty estimate of FFs as provided by Ref. 22] already accounts for the errors of the SIDIS data and that of the particular PDF set employed in their extraction. This implies a certain degree of double counting of uncertainties. On the other hand, excluding the error on FFs altogether would disregard legitimate sources of uncertainties in the reweighting procedure, such as those coming from other, non-SIDIS data sets used in the determination of FFs. Of course, this conceptual problem would not be present in a simultaneous global analysis of PDFs and FFs.

In Fig. 4 we show the $\chi^{2} / N_{\text {data }}$ distribution of the PDF replicas before and after their reweighting (upper and lower left panels, respectively), the scale factor $\mathcal{P}(\alpha)$ probability (lower right panel) and the distribution of weights $w_{k}$ (upper right panel), for three different options regarding the FFs contribution to the $\chi^{2}$. The first one consists of neglecting the FFs uncertainty entirely, which
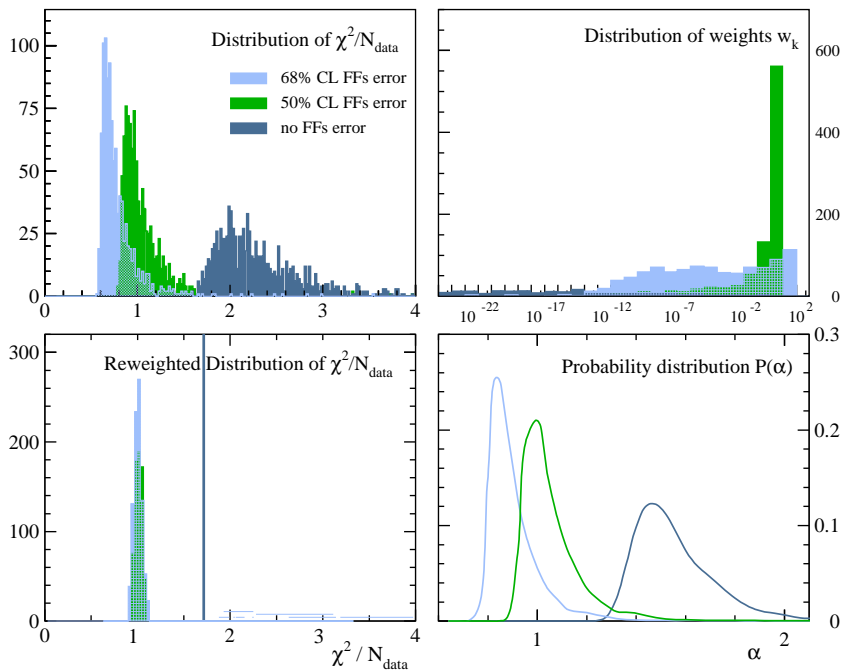

FIG. 4: Distribution of $\chi^{2}$ per data point for the combined kaon multiplicity data sets of HERMEs and Compass before and after reweighting, upper and lower left panel, respectively, the weights $w_{k}$ (upper right panel) and of $\mathcal{P}(\alpha)$ (lower right panel). Each distribution is computed neglecting the error coming from the FFs (green), including the $68 \%$ C.L. estimates of Ref. 22] (blue), and an intermediate C.L. value adjusted such that $\mathcal{P}(\alpha)$ peaks at one (red); see text.

leads to a $\chi^{2} / N_{\text {data }}$ distribution and a $\mathcal{P}(\alpha)$ peaked at values much larger than one. This is a clear indication for underestimated errors or and inadequate theory, and, in fact, the effective number of surviving replicas is $\sim 1$. For the other extreme, including the full $68 \%$ C.L. uncertainties as estimated in [22], yields peaks below unity, characteristic of overestimated errors; another unwanted result in applying the reweighting method. The third option amounts to adjusting the C.L. such that $\mathcal{P}(\alpha)$ is peaked at unity as one would expect in a consistent reweighting of PDFs. The effective number of surviving replicas for this choice is found to be 310 . In the following, we thus apply this criterion to include the uncertainties of FFs when reweighting PDFs based on SIDIS data.

\section{RESULTS OF THE REWEIGHTING EXERCISE}

In this section we present and discuss the outcome of the reweighting exercise based on kaon multiplicities in SIDIS in terms of the resulting set of PDFs and combinations of PDFs that quantify best the breaking of charge and flavor symmetry we are interested in. We also assess the impact of FFs in the reweighting process, and their refinement through the iterative procedure outlined above.

Let us start with the strange quark density which is 
the main objective of our reweighting exercise with kaon multiplicities in SIDIS. In the upper left panel of Fig. [5we show different, recent strange quark PDFs as a function of $x$ as obtained by MMHT 14 33] (red line), CT 14 [34] (green line) and NNPDF 3.0 [21] (black line) to which all results are normalized. Also included is the uncertainty band of the NNPDF 3.0 analysis (light grey band). The first striking feature to notice in the plot are the differences between the current PDFs analyses for the strange quark distribution which can exceed $40 \%$ in case of MMHT 14 and even lie outside of the NNPDF3.0 uncertainty band in some region of $x$.

To demonstrate the impact of the Hermes and COMPASS kaon multiplicities, the light blue curve and the blue shaded band represent the result and the uncertainty estimate of a first reweighting of the NNPDF 3.0 replicas for the strange quark PDF. This reweighting is performed by combining the replicas with the DSS 17 set of kaon FFs to compute the weights $w_{k}$ by comparing to the SIDIS data. One should recall that the DSS 17 global analysis makes use of the MMHT 14 set of PDFs and their uncertainty estimates to determine the parton-to-kaon FFs. We find that this exercise leads to a strange quark PDF that is in between the starting set, i.e. NNPDF 3.0, and the MMHT 14 set that is utilized by DSS 17. The corresponding uncertainty band is significantly smaller than that provided by the original NNPDF 3.0 analysis, indicating that a large number of the NNPDF 3.0 replicas are excluded by the HERMES and Compass kaon multiplicity data.

The above mentioned sizable differences between the strange quark densities in recent PDF sets obviously questions the use of any particular PDF set in the extraction of FFs, MMHT 14 in case of the recent DSS 17 global fit. It even casts doubts on any result that is obtained by adopting these FFs. In order quantify and even avoid this potential bias from the use of a specific set of PDFs inherent in the DSS 17 analysis of FFs, we performed a second reweigthing exercise with the SIDIS kaon multiplicities. To this end, we take the result from the first reweighting as the input PDF set for a reanalysis of kaon FFs, following the framework of DSS 17 in all other aspects. The so obtained set of FFs is then used for another round of reweighting of the NNPDF 3.0 replicas. The outcome of this iterative procedure is the light blue and black dashed line also shown in upper left panel of Fig. 5. As can be seen, the iteration os now much closer to the original strange quark density of NNPDF 3.0 but, again, with smaller uncertainties (hatched band). Notice that the error band resulting from one iteration of the reweighting procedure is different from the one without iteration of the FFs, because in the comparison to data the improved set of FFs penalizes the NNPDF 3.0 replicas in the ensemble differently.

The results of these exercises suggest that the HeRmes and Compass kaon multiplicity data appear to favor a PDF set more similar to NNPDF 3.0 than to the other current sets, CT 14 and MMHT 14. Indeed, the $\chi^{2}$ of the

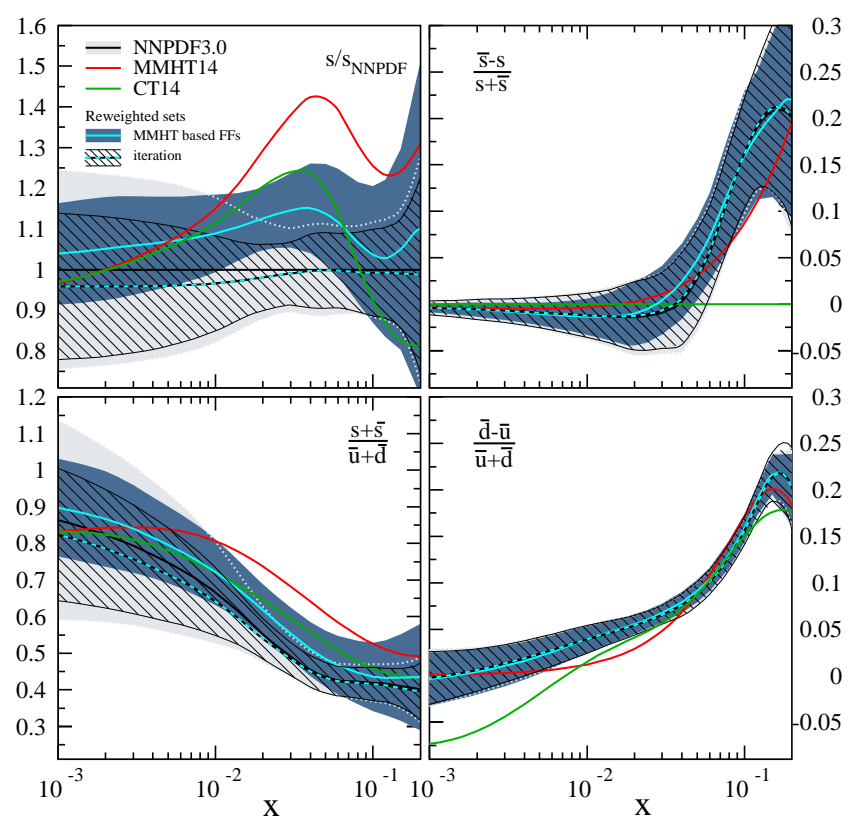

FIG. 5: Reweighting of the strange quark distribution (upper left panel) and for the PDF combinations sensitive to charge (upper right panel) and flavor (lower panels) symmetry breaking using the DSS 17 set of kaon FFs that is based on the MMHT 14 set of PDFs; see text. The dashed light blue and black lines and the hatched areas represent the results of one iteration of the reweighting procedure and the corresponding uncertainty bands, respectively; see text. All results are shown at a scale of $Q^{2}=5 \mathrm{GeV}^{2}$.

newly obtained FFs based on the reweighted set of PDFs is significantly smaller, 1041.3 units, than the original one, 1271.7, obtained in the DSS 17 fit [22], largely due to an improved description of the HERMES and COMPASS data. We therefore conclude that the reweighting of the PDFs using SIDIS multiplicity data has a significant impact with regard to the strange quark distribution and, in turn, the extraction of kaon FFs. We also wish to stress that even though the first reweighting procedure seems to be biased towards the MMHT 14 set of PDFs through the use of the DSS $17 \mathrm{FFs}$, the exercise helps to show that the method, in principle, allows for a significant departure from the mean value of ensemble of replicas one starts from.

The upper right panel of Fig. [5illustrates, in the same way as before, the strange quark-antiquark or charge asymmetry, which is rather small for all the different sets of PDFs in most of the $x$ range, except at rather large values. As can be seen, in this case the result of the reweighting is not as significant as for the strange quark PDF both in terms of a change in the asymmetry or in a reduction of the uncertainty band. This clearly suggest a much weaker grip of the Hermes and Compass data on this quantity beyond to what is already included in current global extractions of PDFs. 
On the contrary, but perhaps not too surprisingly, a larger impact of the reweighting is again found for the ratio between strange and non-strange light sea quarks which is shown in the lower left panel of Fig. 5. The ratio measures the degree of $\mathrm{SU}(3)$ breaking in the proton and has been the subject of considerable interest over the past decades. Our result confirms a rather strong $x$ dependence in the strangeness suppression, that weakens, i.e., the ratio approaches unity, towards smaller momentum fractions. As for the strange quark distribution, the iterated reweighting procedure favors the ratio obtained with the NNPDF 3.0 set of PDFs, but slightly modifies it and reduces the corresponding uncertainty.

As one anticipates, the reweigthing procedure using the Hermes and Compass kaon multiplicity data has very little impact on the difference between the $\bar{u}$ and $\bar{d}$ antiquarks as can be inferred form the lower right panel of Fig. [5. SIDIS multiplicities for kaons are not competitive with the constraints on $\bar{u}$ and $\bar{d}$ already included in all the PDF fits.

For completeness, Fig. 6 presents the results of the reweighting exercises with and without iteration, performed in the same way as discussed above, for the $u$, $d, \bar{u}$, and $\bar{d}$ quarks. For all these distributions, differences among the different, current sets of PDFs are much smaller to begin with (at most at a level of 10\%), i.e., the light quark flavors are already much better constrained than the strange quark PDF. Even though the charged kaon multiplicities depend strongly on the $u$ and $\bar{u}$ PDFs, and, in principle, the reweighting could have a

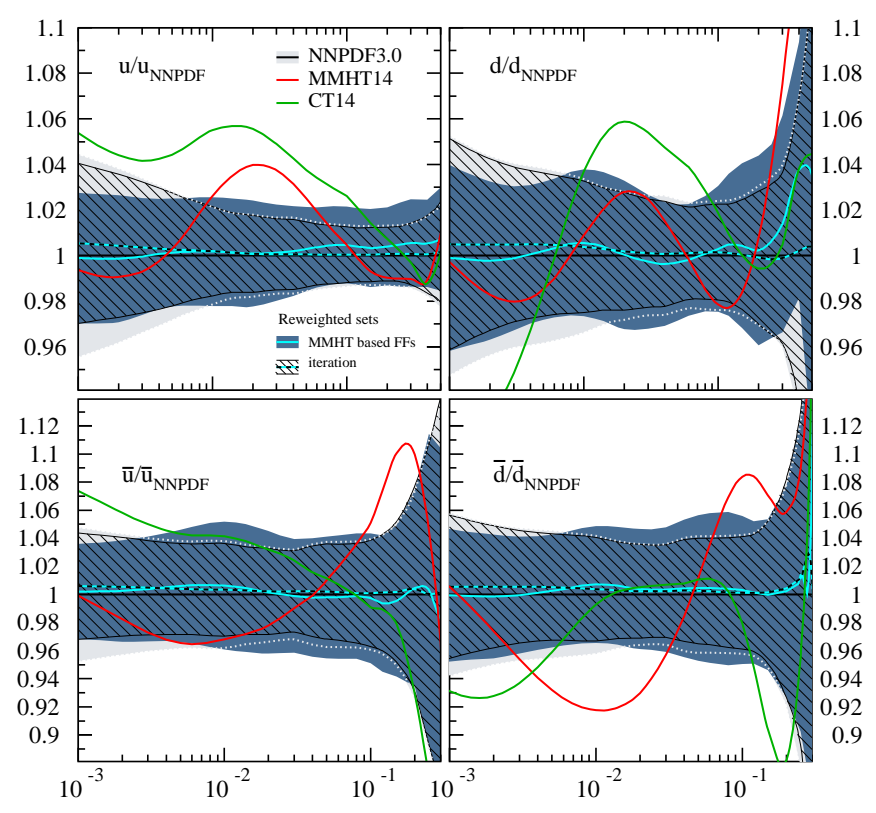

FIG. 6: Same as Fig. 5 but now for the non-strange light quark PDF flavors, again normalized to the corresponding mean value of the NNPDF 3.0 replicas.

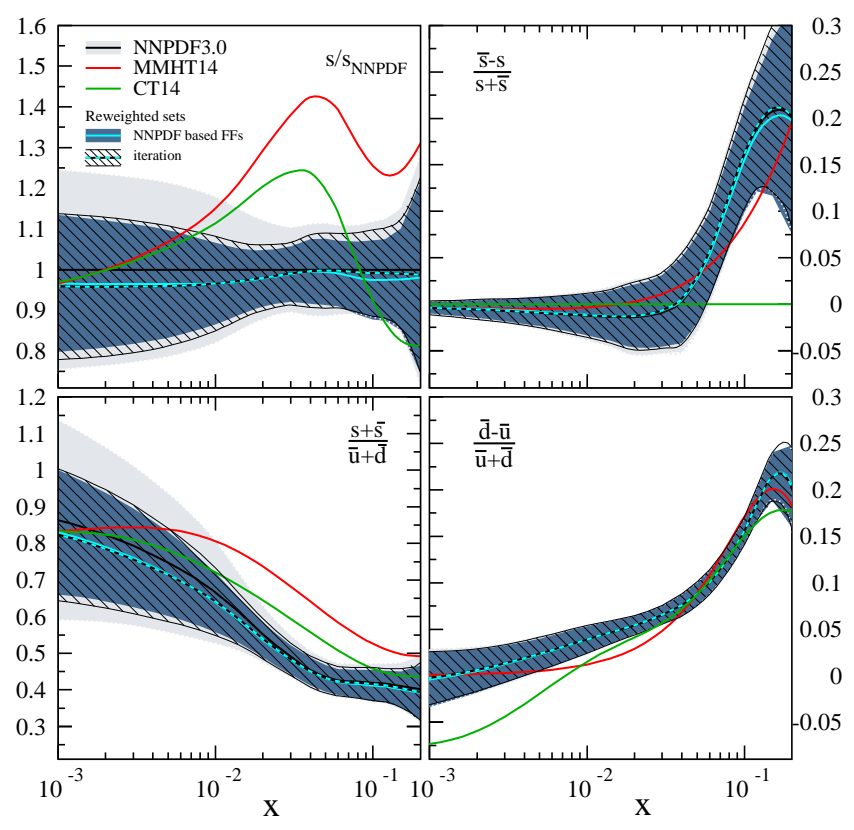

FIG. 7: Same as Fig. 5 but now starting from a variant of the DSS 17 set of kaon FFs, extracted using the NNPDF 3.0 PDF set as input; see text.

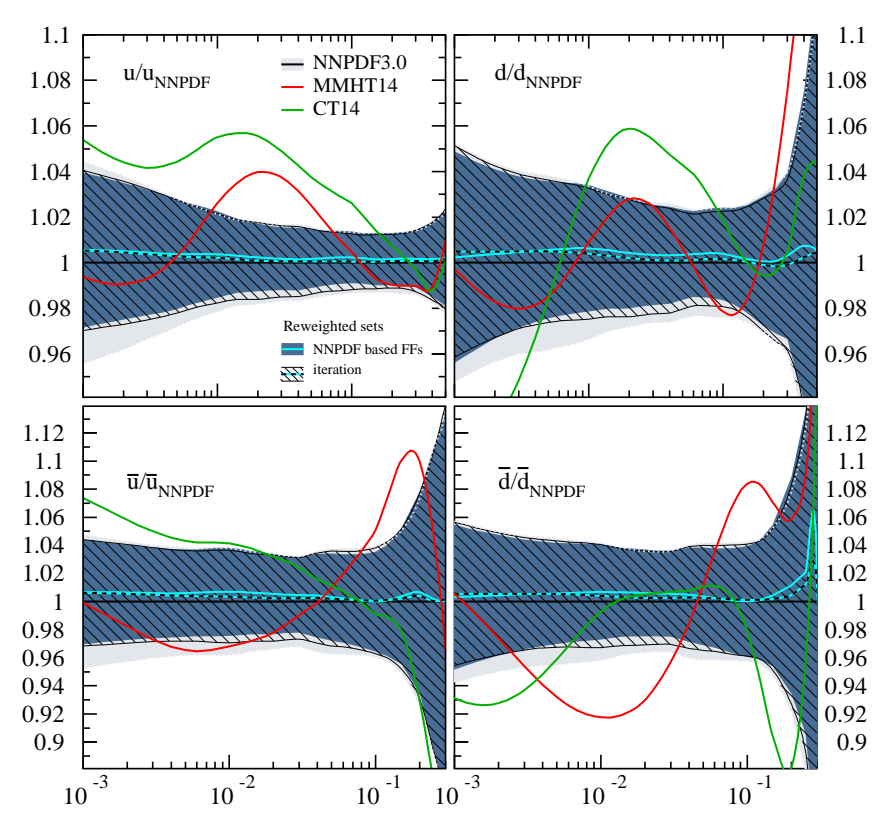

FIG. 8: Same as Fig. 6 but now starting from a variant of the DSS 17 set of kaon FFs, extracted using NNPDF 3.0 PDF set as input; see text.

non-negligible impact, it turned out that is has essentially none.

Finally, in Figs. 7 and 8 we repeat the reweighting ex- 
ercises performed above but now with a set of kaon FFs that was obtained from a DSS 17-like global analysis using the NNPDF 3.0 set of PDFs as input. In this case, the results of the first reweigthing (light blue curves) already resemble those obtained after the first iteration in Figs. 5 and 6 above. More importantly, the results now change only in a negligible way upon iteration (light blue and black dashed line). This highlights the robustness of the proposed iterative reweighting method and the rapid convergence to the same results despite starting from different sets of FFs. The $\chi^{2}$ of the global fit of FFs in this approach, 1025.7 units, only changes slightly from the one obtained above.

We note that recently, the simultaneous extraction of spin-dependent PDFs and FFs was studied in Ref. 35. using an iterative Monte-Carlo method. The main objective of their analysis was the helicity strangeness PDF. The precise data on unpolarized SIDIS multiplicities and the crucial bias from unpolarized PDFs on the extraction of FFs are both not addressed in this paper.

\section{CONCLUSIONS AND OUTLOOK}

SIDIS multiplicities depend crucially on parton distribution functions, and, therefore, precise measurements of them should in principle constrain significantly PDFs in global analyses. In practice, any use of SIDIS multiplicities also requires the best possible knowledge of partonto-hadron fragmentation functions, whose determination in turn also involves PDFs. Because of this complication, combined extractions of parton density and fragmentation functions have been suggested since the early days of the parton model. The rather cumbersome technical details of such simultaneous global QCD fits have prevented so far their realization and implementation.

To circumvent these complications, we have proposed an iterative strategy that is based on the reweighting approach, already routinely utilized for parton densities. As a case study, we have implemented this procedure to analyze the combined impact of recent charged kaon multiplicity data obtained in deep-inelastic scattering on parton density and fragmentation functions. It was demonstrated that the method converges very fast to a stable result for new optimal sets of these non-perturbative functions.

Our study has revealed a significant impact of the kaon multiplicities on the strange quark distribution and its uncertainties as well as on the ratio between strange and light non-strange sea quarks in the proton. In the kinematic regime relevant for the analyzed HERMES and Compass multiplicity data, we find that parton densities close to the NNPDF 3.0 set yield the optimum description of the data in terms of $\chi^{2}$.

The recent availability of comparatively more precise charged pion multiplicities and parton-to-pion fragmentation functions [36], and, eventually, extremely precise SIDIS data in a much wider kinematical range at a possible future Electron Ion Collider [37], highlights the increasing relevance of this kind of combined extractions of parton density and fragmentation functions, both of them pillars of the perturbative QCD description of hard processes based on the notion of factorization.

\section{Acknowledgments}

We are grateful to Juan Rojo for his encouraging support and help and to G. Schnell, E.C. Aschenauer (HERMES) and F. Kunne, E. Seder (Compass) for helpful discussions about their SIDIS measurements. This work was supported in part by CONICET and ANPCyT.
[1] See, e.g., J. Butterworth et al., J. Phys. G 43, 023001 (2016).

[2] See, e.g., A. Metz and A. Vossen, Prog. Part. Nucl. Phys. 91, 136 (2016).

[3] C. Alexandrou and K. Jansen, Nucl. Part. Phys. Proc. 261-262, 202 (2015).

[4] See, e.g., T. Gutsche, V. E. Lyubovitskij, and I. Schmidt, Eur. Phys. J. C 77, 86 (2017).

[5] J. Rojo et al., J. Phys. G 42, 103103 (2015) doi:10.1088/0954-3899/42/10/103103

[6] G. Aad et al. [ATLAS Collaboration], Phys. Rev. Lett. 109, 012001 (2012).

[7] S. Chatrchyan et al. [CMS Collaboration], Phys. Rev. D 90, 032004 (2014).

[8] M. Aaboud et al. [ATLAS Collaboration], Eur. Phys. J. C 77, 367 (2017).

[9] A. O. Bazarko et al. [CCFR Collaboration], Z. Phys. C 65, 189 (1995). hep-ex/9406007.

[10] M. Goncharov et al. [NuTeV Collaboration], Phys. Rev.
D 64, 112006 (2001)

[11] D. Mason et al. [NuTeV Collaboration], Phys. Rev. Lett. 99, 192001 (2007).

[12] O. Samoylov et al. [NOMAD Collaboration], Nucl. Phys. B 876, 339 (2013).

[13] A. Airapetian et al. [Hermes Collaboration], Phys. Rev. D 87, 074029 (2013).

[14] See, e.g., W. C. Chang and J. C. Peng, Prog. Part. Nucl. Phys. 79, 95 (2014).

[15] R. P. Feynman, "Photon-Hadron Interactions", Reading, 1972.

[16] C. Adolph et al. [Compass Collaboration],, Phys. Lett. B 767, 133 (2017).

[17] R. D. Ball et al. [NNPDF Collaboration], Nucl. Phys. B 849, 112 (2011) Erratum: [Nucl. Phys. B 854, 926 (2012)] Erratum: [Nucl. Phys. B 855, 927 (2012)],

[18] R. D. Ball et al., Nucl. Phys. B 855, 608 (2012).

[19] N. Armesto, J. Rojo, C. A. Salgado, and P. Zurita, JHEP 1311, 015 (2013). 
[20] H. Paukkunen and P. Zurita, JHEP 1412, 100 (2014).

[21] R. D. Ball et al. [NNPDF Collaboration], JHEP 1504, 040 (2015).

[22] D. de Florian, M. Epele, R. J. Hernandez-Pinto, R. Sassot, and M. Stratmann, Phys. Rev. D 95, 094019 (2017).

[23] D. de Florian, R. Sassot, and M. Stratmann, Phys. Rev. D 75, 114010 (2007).

[24] G. Agakishiev et al. [Star Collaboration], Phys. Rev. Lett. 108 (2012) 072302.

[25] B. Abelev et al. [Alice Collaboration], Phys. Lett. B 736, 196 (2014).

[26] M. Leitgab et al. [Belle Collaboration], Phys. Rev. Lett. 111, 062002 (2013).

[27] J. P. Lees et al. [BABAr Collaboration], Phys. Rev. D 88, 032011 (2013).

[28] D. Buskulic et al. [Aleph Collaboration], Z. Phys. C 66, 355 (1995).

[29] P. Abreu et al. [DelPhi Collaboration], Eur. Phys. J. C $\mathbf{5}, 585$ (1998).

[30] R. Akers et al. [OpAl Collaboration], Z. Phys. C 63, 181 (1994).

[31] K. Abe et al. [Shd Collaboration], Phys. Rev. D 59, 052001 (1999).

[32] G. Abbiendi et al. [OpAL Collaboration], Eur. Phys. J. C 16, 407 (2000).

[33] L. A. Harland-Lang, A. D. Martin, P. Motylinski, and R. S. Thorne, Eur. Phys. J. C 75, 204 (2015).

[34] J. Gao et al., Phys. Rev. D 89, 033009 (2014).

[35] J. J. Ethier, N. Sato, and W. Melnitchouk, arXiv:1705.05889 [hep-ph].

[36] D. de Florian, R. Sassot, M. Epele, R. J. HernandezPinto, and M. Stratmann, Phys. Rev. D 91, 014035 (2015).

[37] A. Accardi et al., Eur. Phys. J. A 52, 268 (2016). 